\title{
Large-scale calculations of nuclear-structure data for simulation data bases
}

\author{
P Möller ${ }^{1, a}$, R. Bengtsson ${ }^{2}$, K.L. Kratz ${ }^{3}$, and H. Sagawa ${ }^{4}$ \\ 1 Theoretical Division, Los Alamos National Laboratory, Los Alamos, NM 87545, USA \\ 2 Department of Mathematical Physics, Lund Institute of Technology, 22100 Lund, Sweden \\ 3 Max-Planck Institut für Chemie, Otto-Hahn Institut, 55128 Mainz, Germany \\ ${ }^{4}$ Center for Mathematical Sciences, University of Aizu, Aizu-Wakamatsu, Fukushima 965-8580, Japan
}

\begin{abstract}
In the macroscopic-microscopic model we have (1) enhanced our FRDM (1992) model of nuclear groundstate masses and shapes, (2) performed a global calculation of nuclear shape isomers, in which we characterize the ground-state and isomer minima in terms of their relative energies and shapes, and furthermore provide the saddle heights between all pairs of minima, and (3) performed a calculation of fission potential-energy surfaces for more than 5 million different shapes for each of 5254 nuclei from $A=170$ to $A=330$. We use an immersion technique borrowed from geography to determine saddle points and minima in these surfaces. We also use this technique to establish if structures such as deep valleys separated by high ridges are present. These would then give rise to different modes of fission.
\end{abstract}

\section{Introduction}

In the macroscopic-microscopic method the starting point of a calculation is usually a determination of the nuclear potential energy for a specific shape. The calculation involves several steps:

1. A shape is prescribed.

2. A single-particle potential with this shape is generated. A spin-orbit term is included.

3. The Schrödinger equation is solved for this deformed potential and single-particle levels and wave-functions are obtained.

4. The shell correction is calculated by use of Strutinsky's method.

5. The pairing correction is calculated in the BCS or LipkinNogami method.

To obtain the total potential energy a macroscopic contribution is added to the shell-plus-pairing correction. The macroscopic potential energy is the sum of a Coulomb energy, a surface energy incorporating the finite range of the nuclear force, and other terms. Full details are found in ref. [1].

Ground-state masses are determined by locating the lowest energy minimum in regions of modest deformation. A fission barrier is the optimum energy trajectory between the ground state and separated fission fragments. To determine this trajectory it is necessary to calculate the nuclear potential energy for a large number of different shapes. In the calculation here we calculate for each nucleus the energy for 5003325 different shapes. Since our model is microscopic and we obtain nuclear wave functions it is possible to calculate a large number of quantities microscopically, for example energy levels, $\beta$-decay transition rates from specific parent configurations to specific daughter configurations, and $\beta$-delayed fission and neutronemission rates.

\footnotetext{
${ }^{a}$ Presenting author, e-mail: moller@lanl.gov
}

\section{Nuclear ground-state masses and deformations}

A goal for a theory of nuclear masses is that it be able to accurately predict masses of nuclei for which no measured values are available. Data for unknown nuclei are needed in many simulations; one example is simulations of the r-process. Our FRDM (1992), published in 1995 [1], was adjusted to a 1989 data base of nuclear masses. In 1997 [2] we compared the published theoretical masses to masses that were not available in the 1989 evaluation but listed in a new mass evaluation [3, 4]. The model error for the 217 predicted masses was comparable to the error relative to the 1989 data base from which the model parameters were determined, cf. figure 1 . We now have a data base of 529 "new" masses available in the most recent Audi evaluation [5]. The error of our published mass table with respect to this whole data set is $0.46 \mathrm{MeV}$, much smaller than with respect to the 1989 data base, cf. figure 2 . In many cases we cannot make exactly the same comparison for other microscopic models because most such models have been adjusted to more recent experimental mass data sets. But although the HFB8 mass model [6] was adjusted to most of the mass data in the Audi 2003 evaluation, we have in figure 3 compared the HFB8 mass table to the same data we used in figure 2. We observe that the HFB8 model error for these nuclei is $0.635 \mathrm{MeV}$. Clearly, this test of the predictive power of the FRDM (1995) is reassuring. An interesting observation is that some points on the proton-rich side of $\beta$-stability indicating large deviations between theory and experiment in figure 1 are not present in figure 2. Since these two figures are based on the identical theory, the difference means that the experimental data points were either removed from the evaluated data base, or changed by more than the error bars. So some of the improvement between figures 1 and 2 occurred because the experimental data changed, not the theory! It is also of interest to note that whereas figure 1 represents a double-blind test (experiment and theory were not aware of 
Table 1. FRDM (1992) and successive enhancements, compared to different data sets. The first column indicates a model designation, the second which data set the model was adjusted to, the third which data set the mass table is compared to, and the last two columns the mean deviation and the model error. Column 4 is the number of nuclei in the data set the model is compared to; the corresponding error is in the last column. A1989, A2003, and A8903 stand for the Audi 1989 mass evaluation [11], the Audi 2003 mass evaluation [5], and masses that are in the 2003 evaluation but not in the 1989 evaluation ("new" masses), respectively. The model constants are given in the middle section. The top line gives the original model constants [1].

\begin{tabular}{|c|c|c|c|c|c|c|c|c|c|c|c|c|c|}
\hline Model & Adj. & Comp. & $\begin{array}{c}N_{\text {nuc }} \\
(\mathrm{MeV})\end{array}$ & $\begin{array}{c}a_{1} \\
(\mathrm{MeV})\end{array}$ & $\begin{array}{c}a_{2} \\
(\mathrm{MeV})\end{array}$ & $\begin{array}{c}J \\
(\mathrm{MeV})\end{array}$ & $\underset{(\mathrm{MeV})}{Q}$ & $\begin{array}{c}a_{0} \\
(\mathrm{MeV})\end{array}$ & $\begin{array}{c}c_{\mathrm{a}} \\
(\mathrm{MeV})\end{array}$ & $\begin{array}{c}C \\
(\mathrm{MeV})\end{array}$ & $\gamma$ & $\begin{array}{c}\mu_{\mathrm{th}} \\
(\mathrm{MeV})\end{array}$ & $\begin{array}{l}\sigma_{\mathrm{th} ; \mu=0} \\
(\mathrm{MeV})\end{array}$ \\
\hline (92) & A1989 & A1989 & 1654 & 16.247 & 22.92 & 32.73 & 29.21 & 0.00 & 0.436 & 60 & 0.831 & 0.0156 & 0.6688 \\
\hline (92) & A1989 & A8903 & 529 & & & & & & & & & 0.1755 & 0.4617 \\
\hline (92) & A1989 & A2003 & 2149 & & & & & & & & & 0.0607 & 0.6314 \\
\hline (92)-a & A1989 & A1989 & 1654 & 16.245 & 23.02 & 32.22 & 30.73 & -2.24 & 0.465 & 104 & 0.927 & 0.0000 & 0.6614 \\
\hline (92)-a & A1989 & A8903 & 529 & & & & & & & & & 0.0174 & 0.4208 \\
\hline (92)-a & A1989 & A2003 & 2149 & & & & & & & & & 0.0114 & 0.6180 \\
\hline (92)-b & A1989 & A1989 & 1654 & 16.286 & 23.37 & 32.34 & 30.51 & -5.21 & 0.468 & 179 & 1.027 & 0.0000 & 0.6591 \\
\hline (92)-b & A1989 & A8903 & 529 & & & & & & & & & 0.0031 & 0.4174 \\
\hline (92)-b & A1989 & A2003 & 2149 & & & & & & & & & 0.0076 & 0.6157 \\
\hline$(06)-a$ & A2003 & A2003 & 2149 & 16.274 & 23.27 & 32.19 & 30.64 & -5.00 & 0.450 & 169 & 1.000 & 0.0000 & 0.6140 \\
\hline (06)-b & A2003 & A2003 & 2149 & & & & & & & & & 0.0191 & 0.6042 \\
\hline (06)-c & A2003 & A2003 & 2149 & 16.274 & 23.27 & 32.18 & 30.59 & -4.84 & 0.45 & 161 & 0.992 & 0.0000 & 0.6038 \\
\hline
\end{tabular}

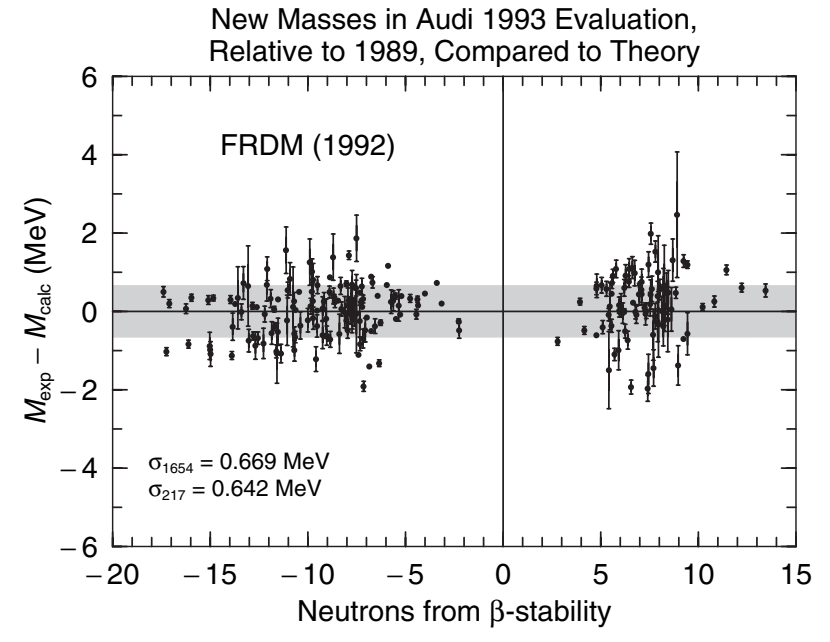

Fig. 1. An early study [2] of the reliability of the FRDM (1992) with respect to 217 subsequently measured masses in ref. [3].

the other) figure 2 represents only a single-blind test (theory was blind, experiment was aware of theory).

\subsection{Enhanced FRDM mass table}

We have now improved the FRDM (1992) mass table. Successive improvements are listed in table 1 . The first three lines show the published FRDM (1992) compared to the data set to which it was adjusted (A1989), new masses measured since then (A8903) and the totality of masses in the most recent mass evaluation (A2003). Because of a 100000 fold increase in computer power since the FRDM (1992) calculation was carried out we can now considerably refine the calculation. On line 4 we show the result of a better optimization of the constants to the 1989 data set. An indication that the original adjustment was not quite optimum is that the mean error $\mu_{\text {th }}$

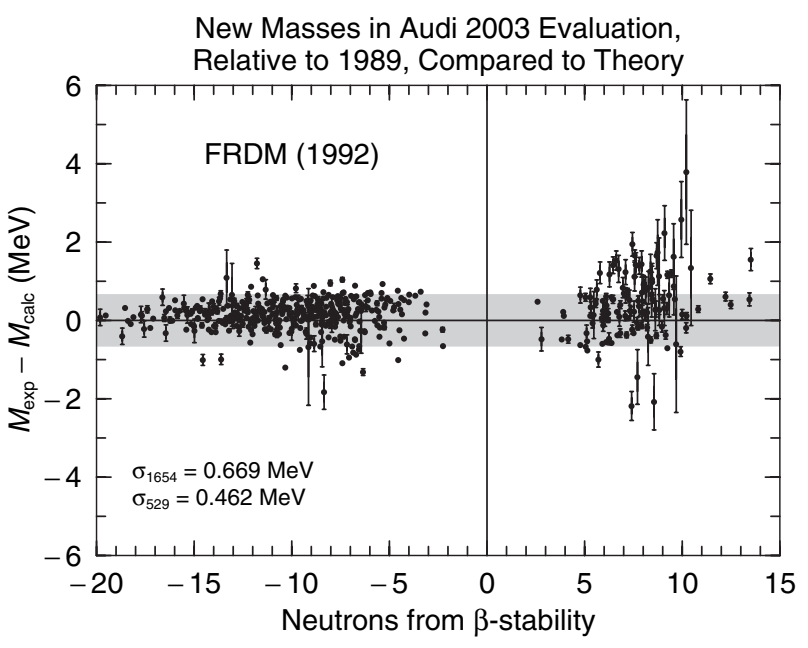

Fig. 2. Reliability of the FRDM (1992) with respect to 529 subsequently measured masses in ref. [5], the most recent evaluation available.

is substantially different from zero, namely $0.0156 \mathrm{MeV}$. In all subsequent adjustments in our investigation here the mean error is zero to four significant digits. Line 5 compares this better optimized model to the 529 new masses. It is interesting to note that the better optimized model has better predictive power $(0.42 \mathrm{MeV}$ versus $0.46 \mathrm{MeV})$ ! Line 6 compares to the entire 2003 data set. The next three lines differ only in that fission barriers are not included in the adjustment. We have earlier [8-10] observed that the FRDM should not be applied to fission barriers. There is only a very minor improvement in the model error in the region of adjustment, but it is of interest to observe that the predictive power also improves slightly. The next line (line 10 in table 1 ) shows the effect of adjusting the model parameters to the newest 2003 data set. The model is extraordinarily stable, the model error only improves by $0.0017 \mathrm{MeV}$. Line 11 shows the effect of triaxial shape degrees 


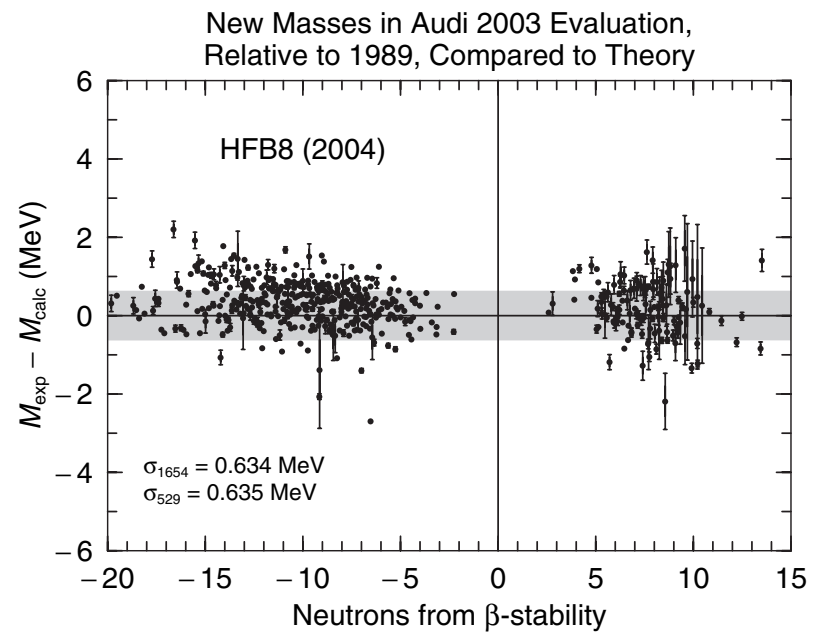

Fig. 3. HFB 8 masses compared to the same experimental data set of 529 masses as considered in figure 2.

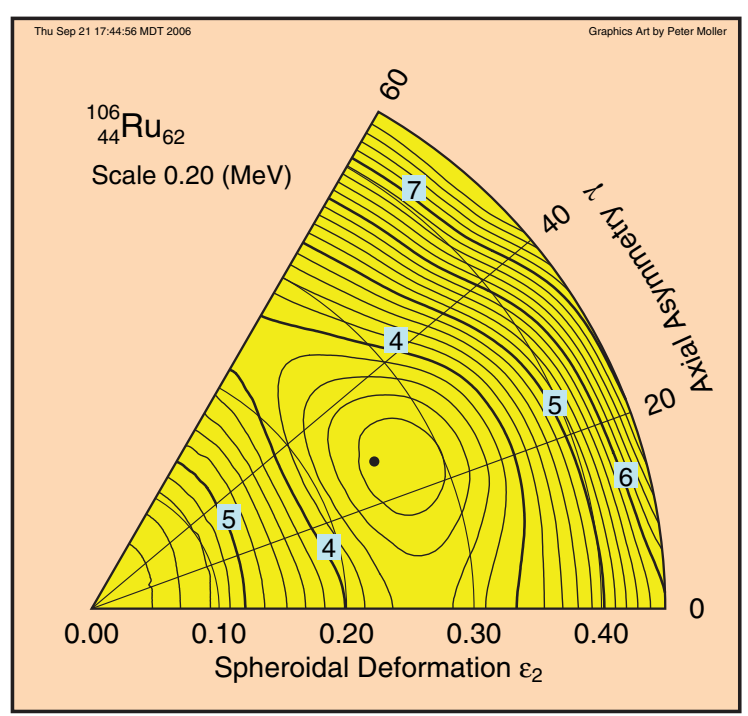

Fig. 4. The calculated ground state shape of ${ }^{106} \mathrm{Ru}$ is triaxial, as is the case for several hundred other nuclei across the nuclear chart, for details see ref. [7].

of freedom on the ground-state masses. Although only about 100 nuclei are affected, cf. figure 4 for an example, the improvement is still $0.01 \mathrm{MeV}$ when averaged over the more than 2000 calculated masses for which experimental data exist. Readjustment of the model parameters after triaxial corrections are included yields a further small reduction in error to $0.6038 \mathrm{MeV}$.

In our 1992 mass calculation [1] the determination of the ground-state mass proceeded in the following way. In the initial step the potential energy was calculated as a function of $\epsilon_{2}$ and $\epsilon_{4}$. The ground-state minimum was identified in this space and the values of the shape coordinates $\epsilon_{2}$ and $\epsilon_{4}$ were tabulated. Then, for each nucleus, two further minimizations were carried out, both with $\epsilon_{2}$ and $\epsilon_{4}$ held fixed at their previously determined values. In one of the minimizations the energy was minimized with respect to $\epsilon_{3}$, in the other $\epsilon_{6}$ was varied (with $\epsilon_{3}=0$ ). The lowest of the two minima obtained was tabulated as the ground state.
Here we considerably refine this calculation. In 1987 , when we performed the first step in our mass calculation, namely the calculation of the potential-energy surfaces versus $\epsilon_{2}$ and $\epsilon_{4}$ we saved (and retained ever since) these simple potential-energy surfaces and a table of all minima found in these surfaces. Now we study the stability of all these minima in a full $4 \mathrm{D}$ deformation space in the coordinates $\epsilon_{2}, \epsilon_{3}, \epsilon_{4}$ and $\epsilon_{6}$. We use a grid with a grid-point distance of 0.01 in all 4 deformation parameters. We start by calculating a 4D "cube" around the minimum found in the $2 \mathrm{D} \epsilon_{2}-\epsilon_{4}$ space. Such a cube consists of 81 grid points, 80 of them on the surface of the cube. The lowest energy will be a grid point on the surface of this cube, unless the initial point determined from the limited $2 \mathrm{D}$ calculation accidentally turns out to be the location of the local minimum. If not, we construct a new 4D cube around the grid-point corresponding to the lowest energy on the surface of the initial cube, taking care not to recalculate energies that are already calculated. We continue in this fashion until the lowest-energy point is not on the surface of the last cube investigated. It is then the interior point in this last cube that is the minimum in the full $4 \mathrm{D}$ space. We investigate all the minima found in the 2D space in this fashion. However, we found that sometimes there exists one minimum for $\epsilon_{3}=0$ and another at $\epsilon_{3} \approx 0.1$ separated by a low ridge. We therefore repeat the search for minima with the above starting points, except $\epsilon_{3}=0.1$ in all the starting points. The lowest of the 4D minima is the optimum choice for the ground state.

This refined calculation improves the accuracy of our original study for two reasons. First, in the original study we only investigated one minimum with respect to higher multipole deformations, namely the lowest minimum found in the $2 \mathrm{D} \epsilon_{2}-\epsilon_{4}$ space. However, if another minimum, a shape isomer, exists in the $2 \mathrm{D} \epsilon_{2}-\epsilon_{4}$ space then, if the effect of varying $\epsilon_{3}$ and $\epsilon_{6}$ is investigated for both these minima, it may turn out that the higher of the original minima becomes the lowest minimum after the full variation of the four deformation parameters. Second, we perform a completely independent variation of the four deformation parameters in a full 4D deformation space, rather than the very restricted variation that was the only tractable computation possible more than 15 years ago. As a historical note, let us mention that the minimization of the potential energy with respect to $\epsilon_{3}$ for fixed $\epsilon_{2}$ and $\epsilon_{4}$ was carried out in 1990 on a set of 6 VAXVMS workstations at the Department of Mathematical Physics in Lund. The calculations were submitted as batch jobs every night (and restarted the subsequent night). It took, at that time, about 1 month to complete these simple calculations. Now, we trivially can access about 100000 times the computer power, which opens up completely new vistas in our investigations. A new mass table based on the 4D calculation is not quite complete, but we can at this stage anticipate a final mass model with an error in the $0.57-0.58 \mathrm{MeV}$ range.

\section{Shape isomers}

In our study of shape isomers we calculate potential energy surfaces for 7206 nuclei in a deformation grid $\varepsilon_{2}=(0.0,0.025, \ldots, 0.45), \gamma=(0.0,2.5, \ldots 60.0)$, and $\varepsilon_{4}=(-0.12,-0.10, \ldots, 0.12)$, altogether 6175 grid points. 


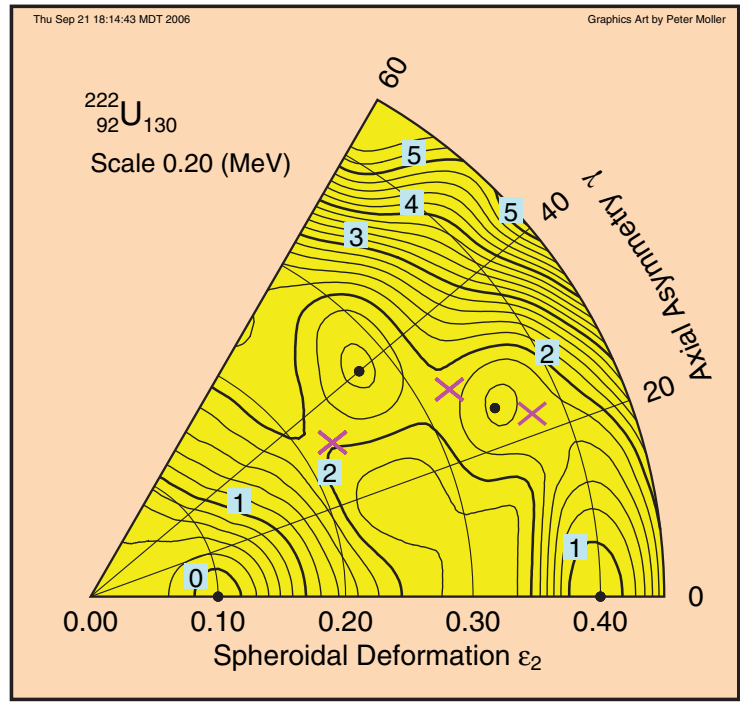

Fig. 5. Quadruple shape coexistence in ${ }^{222} U$. Two of the shapecoexisting minima are triaxial; the ground state is near spherical.

This calculation is described in ref. [7], which focused on the effect of triaxiality on the nuclear ground state. From this calculation we also determine the shape and energy of shape-isomer minima and the saddle heights between all pairs of minima by immersion techniques. A complete table of results and graphs will be submitted to Aтомic DATA AND Nuclear Data Tables [12]. Due to space limitation we can here only present one interesting example: quadruple shape coexistence in ${ }^{222} \mathrm{U}$ shown in figure 5 . The three shape minima are all less than $1.5 \mathrm{MeV}$ above the ground-state at $\epsilon_{2}=0.10$. However, from the 4D calculation above we know that this ground-state is lowered by reflection-asymmetric shape degrees of freedom. In this $4 \mathrm{D}$ space the minimum at $\epsilon_{2}=0.4$ is $2.206 \mathrm{MeV}$ above the ground state. The effect of axial asymmetry and reflection asymmetry on the nuclear ground state will be submitted for publication soon [13].

\section{Fission barriers}

We have calculated fission potential-energy surfaces for 5254 nuclei from $A=170$ to $A=330$. For each nucleus the energy is calculated for 5009325 different shapes in a 5-dimensional deformation space. From these calculations we can determine, by immersion techniques, minima, saddle points between minima, valleys leading to different scission configurations (that is different fission modes), ridge heights between valleys, and the distinctly different saddle points that provide the entry doorway to the different fission modes. Triaxial shapes were studied in the vicinity of the first barrier peak in a 3D calculation in the $\epsilon$ parameterization. In figure 6 we show for each compound system $(Z, N)$ the barrier height minus the neutron separation energy. When this number is negative fission is energetically possible in neutron capture on $(Z, N-1)$. Analysis of the debris after nuclear explosions

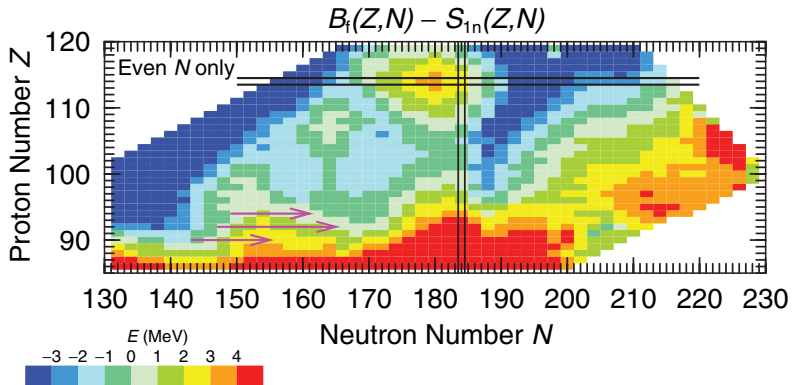

Fig. 6. Calculated barrier height minus neutron-separation energy for the compound system $(Z, N)$. The arrows indicate the observed start and end of observed prompt neutron-capture chains on seed Th, U, and $\mathrm{Pu}$ isotopes in nuclear explosions [14].

shows that capture chains on seed nuclei, indicated by red arrows, stopped at the tips of the arrows [14]. Since the plotted function becomes negative here it is consistent with the observations; according to our calculation capture cannot proceed further. The Th captures end earlier but this is thought to be due to very low capture cross sections early in this chain [14]. The consistency of our calculations with observations far from stability offers encouragement for applications to the r-process region.

\section{References}

1. P. Möller, J.R. Nix, W.D. Myers, W.J. Swiatecki, At. Data Nucl. Data Tables 59, 185 (1995).

2. P. Möller, J.R. Nix, K.-L. Kratz, At. Data Nucl. Data Tables 66, 131 (1997).

3. G. Audi, A.H. Wapstra, Nucl. Phys. A 565, 1 (1993).

4. G. Audi, A.H. Wapstra, Nucl. Phys. A 565, 66 (1993).

5. G. Audi, A.H. Wapstra, C. Thibault, Nucl. Phys. A 729, 337 (2003).

6. M. Samyn, S. Goriely, M. Bender, J.M. Pearson, Phys. Rev. C 70, 044309 (2004).

7. P. Möller, R. Bengtsson, P. Olivius, B.G. Carlsson, T. Ichikawa, Phys. Rev. Lett. 97, 162502 (2006).

8. P. Möller, A. Iwamoto, Phys. Rev. C 61, 047602 (2000).

9. P. Möller, D.G. Madland, A.J. Sierk, A. Iwamoto, Nature 409, 785 (2001).

10. P. Möller, A. J. Sierk, A. Iwamoto, Phys. Rev. Lett. 67, 072501 (2004).

11. G. Audi, Midstream atomic mass evaluation (private communication, 1989), with four revisions.

12. P. Möller, R. Bengtsson, K.-L. Kratz, H. Sagawa, At. Data Nucl. Data Tables (2008, to be published).

13. P. Möller, R. Bengtsson et al., At. Data Nucl. Data Tables (2007, to be published).

14. S.A. Becker, Carnegie Observatories Astrophysics Series, Vol. 4, Origin and Evolution of the Elements, edited by A. McWilliam, M. Rauch (2003), (Pasadena: Carnegie Observatories, URL: http://www.ociw.edu/ociw/symposia/series/symposium4/ proceedings.html). 